\title{
Correction to: Low levels of pyruvate induced by a positive feedback loop protects cholangiocarcinoma cells from apoptosis
}

\author{
Mingming Zhang ${ }^{1,2,4 \dagger}$, Yida Pan ${ }^{3+}$, Dehua Tang ${ }^{1 \dagger}$, Robert Gregory Dorfman ${ }^{5}$, Lei Xu' ${ }^{6}$, Qian Zhou ${ }^{4}$, Lixing Zhou ${ }^{1}$, \\ Yuming Wang ${ }^{1}$, Yang $\mathrm{Li}^{6}$, Yuyao Yin ${ }^{1}$, Bo Kong ${ }^{1,7}$, Helmut Friess ${ }^{7}$, Shimin Zhao ${ }^{2,4}$, Jian-lin Wu ${ }^{8^{*}}$, Lei Wang ${ }^{1^{*}}$ and \\ Xiaoping Zou ${ }^{1 *}$
}

\section{Correction to: Zhang et al. Cell Communication and Signaling (2019) 17:23 https://doi.org/10.1186/s12964-019-0332-8}

Following publication of the original article [1], the authors reported an error in Fig. 3. The corrected Fig. 3 is given below. Please note that these revisions do not affect the overall conclusions reported in the article. The authors apologise for the error and any inconvenience caused.

\section{Author details}

'Department of Gastroenterology, Nanjing Drum Tower Hospital, the Affiliated Hospital of Nanjing University Medical School, Nanjing University, No.321 Zhongshan Road, 210008 Nanjing, People's Republic of China. ${ }^{2}$ Key laboratory of Reproduction Regulation of NPFPC (SIPPR, IRD), Fudan University, Shanghai 200032, China. ${ }^{3}$ Department of Digestive Diseases of Huashan Hospital, Shanghai, China. ${ }^{4}$ School of Life Sciences, Fudan University, Shanghai, China. ${ }^{5}$ Northwestern University Feinberg School of Medicine, Chicago, IL, USA. ${ }^{6}$ Department of Gastroenterology, Nanjing Medical University Affiliated Drum Tower Clinical Medical College, Nanjing Medical University, Nanjing, China. ${ }^{7}$ Department of Surgery, Technical University of Munich (TUM), Munich, Germany. ${ }^{8}$ State Key Laboratory of Quality Research in Chinese Medicine, Macau Institute for Applied Research in Medicine and Health, Faculty of Chinese Medicine, Macau University of Science and Technology, Avenida Wai Long, Taipa, Macao 442000, People's Republic of China.
Received: 24 April 2019 Accepted: 24 April 2019

Published online: 06 May 2019

\section{Reference}

1. Zhang, et al. Low levels of pyruvate induced by a positive feedback loop protects cholangiocarcinoma cells from apoptosis. Cell Commun Signal. 2019;17:23 https://doi.org/10.1186/s12964-019-0332-8.

\footnotetext{
*Correspondence: jlwu@must.edu.mo; 867152094@qq.com;

zouxp@nju.edu.cn

${ }^{\dagger}$ Mingming Zhang, Yida Pan and Dehua Tang contributed equally to this work.

${ }^{8}$ State Key Laboratory of Quality Research in Chinese Medicine, Macau Institute for Applied Research in Medicine and Health, Faculty of Chinese Medicine, Macau University of Science and Technology, Avenida Wai Long, Taipa, Macao 442000, People's Republic of China

${ }^{1}$ Department of Gastroenterology, Nanjing Drum Tower Hospital, the Affiliated Hospital of Nanjing University Medical School, Nanjing University, No.321 Zhongshan Road, 210008 Nanjing, People's Republic of China Full list of author information is available at the end of the article
}

(c) The Author(s). 2019 Open Access This article is distributed under the terms of the Creative Commons Attribution 4.0 International License (http://creativecommons.org/licenses/by/4.0/), which permits unrestricted use, distribution, and reproduction in any medium, provided you give appropriate credit to the original author(s) and the source, provide a link to the Creative Commons license, and indicate if changes were made. The Creative Commons Public Domain Dedication waiver (http://creativecommons.org/publicdomain/zero/1.0/) applies to the data made available in this article, unless otherwise stated. 


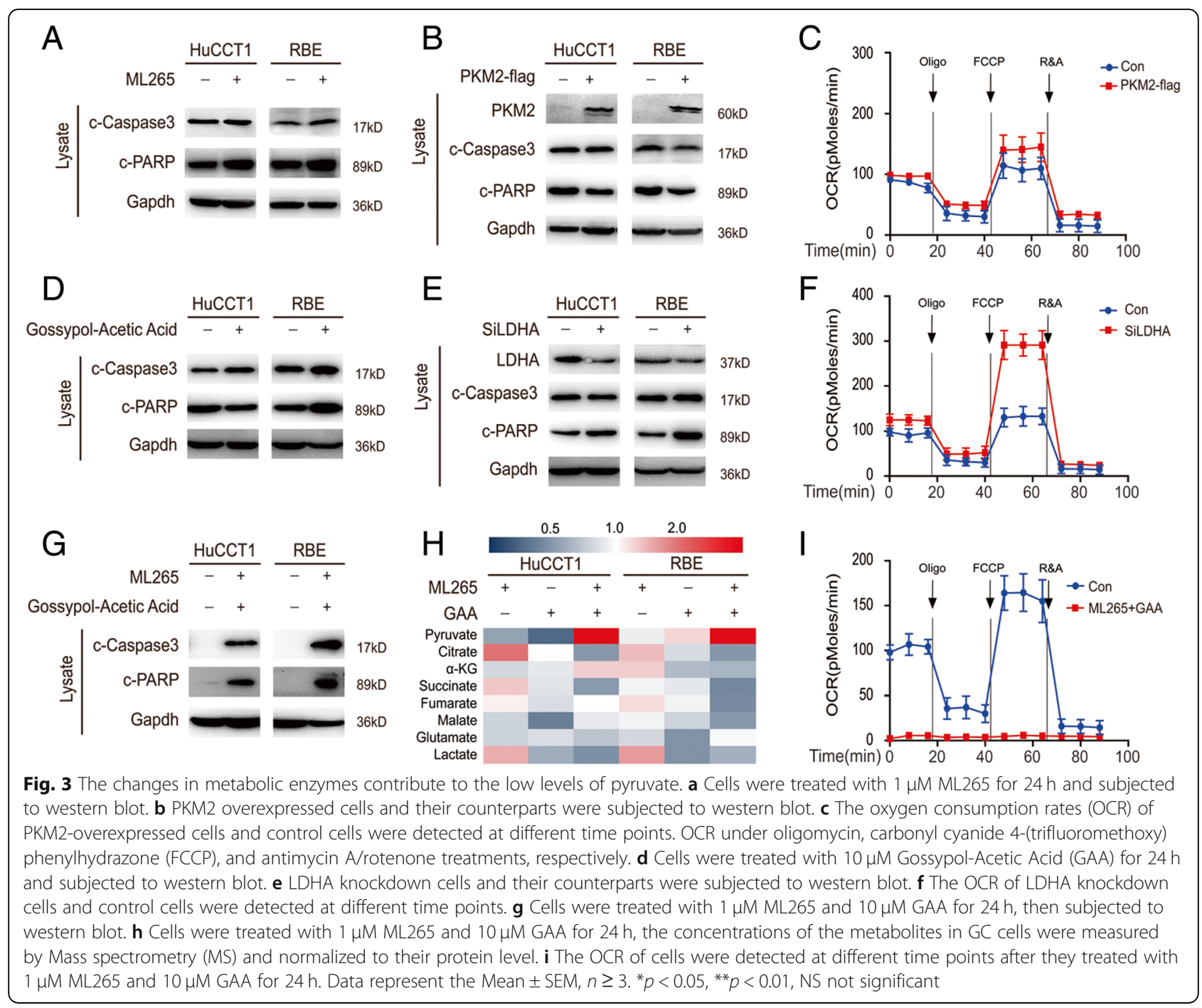

